\title{
Oxidative stress and biochemical markers in prenatally androgenized sheep after neonatal treatment with GnRH agonist
}

This article was published in the following Dove Medical Press journal: Journal of Inflammation Research

Jandui Escariãoda Nóbrega,' Joabel Tonelotto dos Santos, ' Lady K Serrano-Mujica,' Guilherme Bochi, ${ }^{2}$ Rafael Noal Moresco, ${ }^{2}$ Vitor Braga Rissi,' Werner Giehl Glanzner,' Felipe W Langer, ${ }^{3}$ Alfredo Quites Antoniazzi,' Paulo Bayard Dias Gonçalves,' Melissa O Premaor, ${ }^{3}$ Fabio V Comim $^{1,3}$

'Laboratory of Biotechnology and Animal Reproduction - BioRep, Federal University of Santa Maria (UFSM), Santa Maria, RS, Brazil; ${ }^{2}$ Laboratory of Clinical Biochemistry, Department of Clinical and Toxicological Analysis, Federal University of Santa Maria (UFSM), Santa Maria, RS, Brazil; ${ }^{3}$ Department of Clinical Medicine, Federal University of Santa Maria (UFSM), Santa Maria, RS, Brazil
Correspondence: Fabio V Comim Federal University of Santa Maria (UFSM), Av Roraima 1000, Room 1337, Santa Maria, RS, Brazil Tel +55 5532208508 Email fabio.comim@ufsm.br
Background: Disruption of the balance between the production of ROS and their removal through enzymatic and non-enzymatic (antioxidant) processes has been proposed as a new mechanism in the pathology of polycystic ovary syndrome (PCOS). Evidence from animal models of PCOS (prenatally androgenized sheep) has suggested that treatment with insulin sensitizers, but not antiandrogens, can reduce increases in ROS.

Materials and methods: In the present study, we investigated the effects of neonatal treatment with a gonadotropin-releasing hormone (GnRH) agonist (leuprolide acetate) on prenatally androgenized sheep with testosterone propionate to determine its impact on oxidative stress molecules (ferric reducing antioxidant power [FRAP], advanced oxidation protein product [AOPP], nitric oxide [NOx], albumin) at 8, 12, and 18 months of age.

Results: Androgenized ewes (but not leuprolide-treated ewes) showed reduced total cholesterol levels associated with a decrease in the ratio of visceral to subcutaneous adiposity (adjusted to abdominal area) as determined by computed tomography. In androgenized ewes at 12 months of age, an increase in subcutaneous fat and relative decrease in the visceral fat compartment did not affect the expression of REDOX markers. At 18 months of age, however, the levels of NOx metabolites decreased in androgenized animals, but remained close to normal in ewes subjected to neonatal treatment with leuprolide acetate. Other oxidative stress parameters (FRAP, AOPP, albumin) did not vary among groups.

Conclusion: Our results demonstrate that the GnRH agonist leuprolide (as a single dose after birth) had weak effects on markers of the oxidative stress balance.

Keywords: animal model of polycystic ovary syndrome, oxidative stress, gonadotropin-releasing hormone agonist, sheep, metabolism

\section{Introduction}

The REDOX state reflects the balance between the production of ROS and their removal through enzymatic and non-enzymatic (antioxidant) processes. Overproduction of ROS plays a role in the development of common disorders such as diabetes mellitus, dyslipidemia, obesity, chronic obstructive pulmonary disorder, and cancer. ${ }^{1-3}$

Polycystic ovary syndrome (PCOS), one of the most common causes of endocrine infertility, is characterized by reproductive and metabolic disturbances as well as disruption of the REDOX state. ${ }^{4-13}$ Studies conducted in women with PCOS revealed increased oxidant and antioxidant markers in the blood, although the results were inconsistent because of the high heterogeneity of patients (age, body mass index, severity of hyperandrogenism) and tests used to evaluate oxidative stress. ${ }^{13-15}$ 
Animal models are widely used to understand the physiopathology of PCOS and can clarify the potential roles of disruptors in oxidative stress. Classical studies using prenatally androgenized (PA) sheep have replicated several abnormalities described in women with PCOS. Puttabyatappa et al used sheep in their study to explore the impact of prenatal and postnatal interventions with flutamide (antiandrogen) and rosiglitazone (insulin sensitizer), respectively, which demonstrated a partial response to flutamide by reducing protein-bound oxidized tyrosine moieties, 3-nitrotyrosine (NY), an oxidative stress marker, in the plasma. ${ }^{16}$ The levels of antioxidant molecules such as high-molecular adiponectin and the mRNA expression were evaluated in the tissues only and were not affected by flutamide or rosiglitazone. ${ }^{16}$

In rodents, pharmacological intervention has gained attention for protecting or reversing the reproductive phenotype of androgenized rodents. Recent studies demonstrated that administration of a gonadotropin-releasing hormone (GnRH) antagonist (cetrorelix acetate) during gestation (E16-E18.5) to rats treated with anti-Mullerian hormone or GNRH agonist depot (leuprolide acetate) at day 5 after birth in rats treated postnatally with testosterone propionate impaired the development of PCOS features (mainly reproductive) in the adult age..$^{17,18}$

Although leuprolide acetate administration was not shown to alter the REDOX state in PCOS rats, ${ }^{18}$ its effects have not been tested in androgenized sheep, which is considered an animal model of PCOS more similar to humans. Therefore, the aim of the present study was to examine the effects of neonatal treatment with a GnRH agonist on PA sheep with testosterone propionate, looking to determine its impact on biochemical and oxidative stress molecules.

\section{Materials and methods}

\section{Animals}

All procedures were approved by the Institutional Committee for Ethics in Animal Experiments at the Federal University of Santa Maria, RS, Brazil (CEUA-UFSM number 0009/2013). The procedures conformed to the guidelines of the Brazilian National Council of Control of Animal Experimentation, which follows the "Principles of Laboratory Animal Care" established by the National Institutes of Health (Bethesda, MD, USA). For this study, multiparous females aged 3-5 years from Corriedale female sheep (a cross of the Merino and Lincoln breeds) were obtained from a local farmer $\left(30^{\circ}\right.$ $20^{\prime}$ south latitude). The animals' cycles were synchronized and then subjected to artificial insemination using a mix of fresh semen collected from adult rams, as previously described. ${ }^{19}$ Pregnancy was confirmed at 30 days by intravaginal/rectal ultrasonography. The animals were raised in an extensive farming system, with free access to water supplemented with mineral salts, pellet feed ( $1.5 \%$ of live weight), and alfalfa hay. The androgenization protocol included administration of testosterone propionate (Androgenol ${ }^{\mathrm{TM}}$; Hertape Calier, Juatuba, Brazil) $100 \mathrm{mg}$ intramuscularly (i.m.) twice per week to pregnant ewes during days 30-90 of gestation..$^{20}$ No treatment was necessary for controls, as supported by the literature. A group of androgenized female sheep was administered i.m. injection of a GnRH agonist (leuprolide acetate depot $7.5 \mathrm{mg}$ ) before 48 hours of life. All lambs were weaned at 3-4 months and had access to hay ad libitum (plus mineral salts). A group of ewes was euthanized with sodium thiopental at 12 months, including four PA ewes and five controls $(\mathrm{C})$, while the remaining animals were euthanized at 19 months of age. Androgenized ewes exhibited higher levels of serum testosterone at 12 months and showed insulin resistance as defined by intravenous glucose tolerance test and higher disruption of cycles $(66 \%$; data not shown).

\section{Laboratory measurements}

An assay kit (Labtest Diagnostics ${ }^{\circledR}$, Lagoa Santa, Brazil) was used to enzymatically evaluate the levels of total blood cholesterol, high-density lipoprotein-cholesterol (HDL-C), low-density lipoprotein-cholesterol (LDL-C) triglyceride, uric acid, and glucose in the serum. Albumin, which is a scavenger antioxidant molecule, has an intra-assay coefficient of variation of $1.1 \%$. Blood was collected by jugular venipuncture into tubes with and without EDTA (Vacutainer ${ }^{\mathbb{R}}$; BD Biosciences, Franklin Lakes, NJ, USA) and the blood was centrifuged $(2,500 \times g$ for 15 minutes $)$ and stored at $-80^{\circ} \mathrm{C}$ until use.

\section{Oxidative stress markers: ferric-reducing ability of plasma (ferric reducing antioxidant power [FRAP]), advanced oxidation protein product (AOPP) levels in serum, nitric oxide (NOx)}

A colorimetric protocol was employed using a Cobas Mira ${ }^{\circledR}$ automated analyzer (Roche Diagnostics, Basel, Switzerland) to estimate the NOx levels and the AOPP in the serum; as well, the FRAP assay was conducted, as previously described. ${ }^{21-23}$ Samples were processed simultaneously. The intra-assay coefficient of variation was $1 \%-2 \%$ for FRAP, $4 \%$ for AOPP, and $8.3 \%$ for NOx. ${ }^{21-23}$ 


\section{Computed tomography and estimation of abdominal and subcutaneous fat}

Computed tomography (CT) 64 channel (acquisition W:1,800 L:400 bone window; GE Healthcare, Little Chalfont, UK) was performed for ewes at 12 months of age within the first hour after euthanasia. According to the attenuation coefficients obtained by CT, images (region of interest) were classified as fat, muscle, or other tissues. Image processing of slices of $1 \mathrm{~mm}$ was performed using Analyze $12.0^{\circledR}$ software (AnalyzeDirect, Overland Park, KS, USA). ${ }^{24}$ The adipose tissue quantification module from Analyze $12.0^{\circledR}$ was used to determine the segmental (3D) amount of fat, subcutaneous (SC) or visceral, in each animal. For this study, control sheep $(n=5)$ and PA $(n=4)$ sheep were employed.

\section{Statistical analysis}

Statistical analysis was performed using GraphPad Prism 7.0 software (GraphPad, Inc., San Diego, CA, USA). Two groups were compared using Student's $t$-test (if a normal distribution was expected) or Mann-Whitney $U$ test (if an asymmetrical distribution of data was expected). Significance was assumed at $P<0.05$. Two-way ANOVA was used to analyze two independent variables.

\section{Results}

\section{Weight, visceral fat, and biochemical features of control (C), PA, and prenatally androgenized and GnRH-treated (LA) sheep}

Figure 1 shows the biochemical profile of control sheep (C) against PA or prenatally androgenized sheep treated neonatally with leuprolide acetate depot (LA) at 12 and 18 months of age. At 12 months, total cholesterol levels were reduced in PA ewes with a mean $\pm \mathrm{SD}$ of $54.86 \pm 9.2 \mathrm{mg} / \mathrm{dL}$ compared to $\mathrm{C}(68.5 \pm 9.9 \mathrm{mg} / \mathrm{dL}, P<0.001)$; LA sheep $(68.5 \pm 4.9 \mathrm{mg} /$ $\mathrm{dL}$ ) were between $\mathrm{C}$ and PA (Figure 1A). No significant differences were observed in the levels of HDL-C (Figure 1B), LDL-C (Figure 1E), or triglycerides (Figure 1F) among the three groups at 12 months. Basal glucose was very similar in the three groups, with values of $70.1 \pm 12.7,72.8 \pm 8.11$, and $70 . \pm 11.3 \mathrm{mg} / \mathrm{dL}$ in the C, PA, and LA groups, respectively (Figure 1I). The triglyceride-glucose (TyG) index, a marker of insulin resistance and metabolic syndrome, ${ }^{25-28}$ was not changed between the control (C) and androgenized groups (PA or LA). As shown in Figure 1J, there were no significant differences in TYG index values in the $C$ group $(1,362 \pm 392)$, PA group $(1,288 \pm 244.3)$, and LA group $(1,336 \pm 1,206.5)$.
Remarkably, uric acid levels were decreased in PA ewes whose levels at 12 months were $0.51 \pm 0.07 \mathrm{mg} / \mathrm{dL}$, while in C ewes this value was $0.83 \pm 0.33 \mathrm{mg} / \mathrm{dL}$ and in LA ewes it was $0.6 \pm 0.28 \mathrm{mg} / \mathrm{dL}$ (Student's $t$-test $P=0.04 \mathrm{PA}$ vs C; Figure $1 \mathrm{M}$ ). PA sheep showed higher weights than controls at 12 months of age (Figure 2A). These animals exhibited an increased mass of SC depot ( $P=0.03$; Figure $2 \mathrm{G}$ ) estimated by $\mathrm{CT} 3 \mathrm{D}$ analysis of an abdominal segment (Figure 2B), and a similar visceral fat depot (Figure $2 \mathrm{H}$ ) and trend to a lower $\mathrm{SC}$ to visceral ratio (Figure 2I) were observed. Curiously, the ratio of SC to visceral ratio adjusted to the abdominal volume (PA animals showed higher weights) revealed a reduced amount of visceral fat in young PA ewes (Figure 2J).

At 18 months of age (and similar weight), as shown in Figure 2A, the differences in biochemical variables among control (C) and the two androgenized groups (PA and LA) disappeared. Total cholesterol (Figure 1C), HDL (Figure 1D), and LDL (Figure 1G) were similar among the three groups. Basal glucose (Figure 1K) and basal triglyceride (Figure 1H) levels were similar among groups: glucose levels were $66 \pm 6.7$ $\mathrm{mg} / \mathrm{dL}$ in C sheep, $66 \pm 3.7 \mathrm{mg} / \mathrm{dL}$ in PA sheep, and $67.7 \pm 2.5$ $\mathrm{mg} / \mathrm{dL}$ in LA sheep. Triglyceride levels in the serum were $12.7 \pm 2.5,14.33 \pm 3.5$, and $15.67 \pm 2.08 \mathrm{mg} / \mathrm{dL}$ in $\mathrm{C}, \mathrm{PA}$, and LA sheep, respectively; these values were not significantly different $(P=0.08$; Figure $1 \mathrm{H})$. TYG did not change among groups. However, there was an increasing trend in TYG in LA ewes $(1,038 \pm 98.8 \mathrm{mg} / \mathrm{dL})$ vs C ewes $(828 \pm 128.4 \mathrm{mg} / \mathrm{dL}$; Student's $t$-test $P=0.06$ ); the values for PA sheep were intermediate $(953.7 \pm 291.1 \mathrm{mg} / \mathrm{dL}$; Figure 1L). Uric acid levels did not vary among these three groups (Figure 1M). Insulin resistance estimated by the intravenous glucose tolerance test was increased in PA and LA sheep at 16 months of age, as previously reported..$^{29}$

\section{Oxidative stress markers and antioxidant capacity (albumin) in serum and plasma}

The levels of oxidants (AOPP and FRAP), NOx, and molecules with antioxidant function (albumin) from month 8 to 18 of life are shown in Figure 2. The serum levels of AOPP (related to oxidation of proteins) decreased over time and did not differ between the three groups (Figure 2C). At month 18 , the AOPP values were 20.6 $\pm 6.1,24.7 \pm 6.8$, and $14.37 \pm 6.3$ $\mu \mathrm{mol} / \mathrm{L}$ in the C, PA, and LA groups, respectively. Evaluation of FRAP (associated with the reducing ability of plasma) showed similar results among groups. Starting at month 8 , the levels of FRAP in the C, PA, and LA groups were 253.8 \pm 36 , $190.7 \pm 4.1$, and $125 \pm 43 \mu \mathrm{mol} / \mathrm{L}$, respectively. At 12 months of age, FRAP levels in the C, PA, and LA groups were $358.3 \pm 73$, 

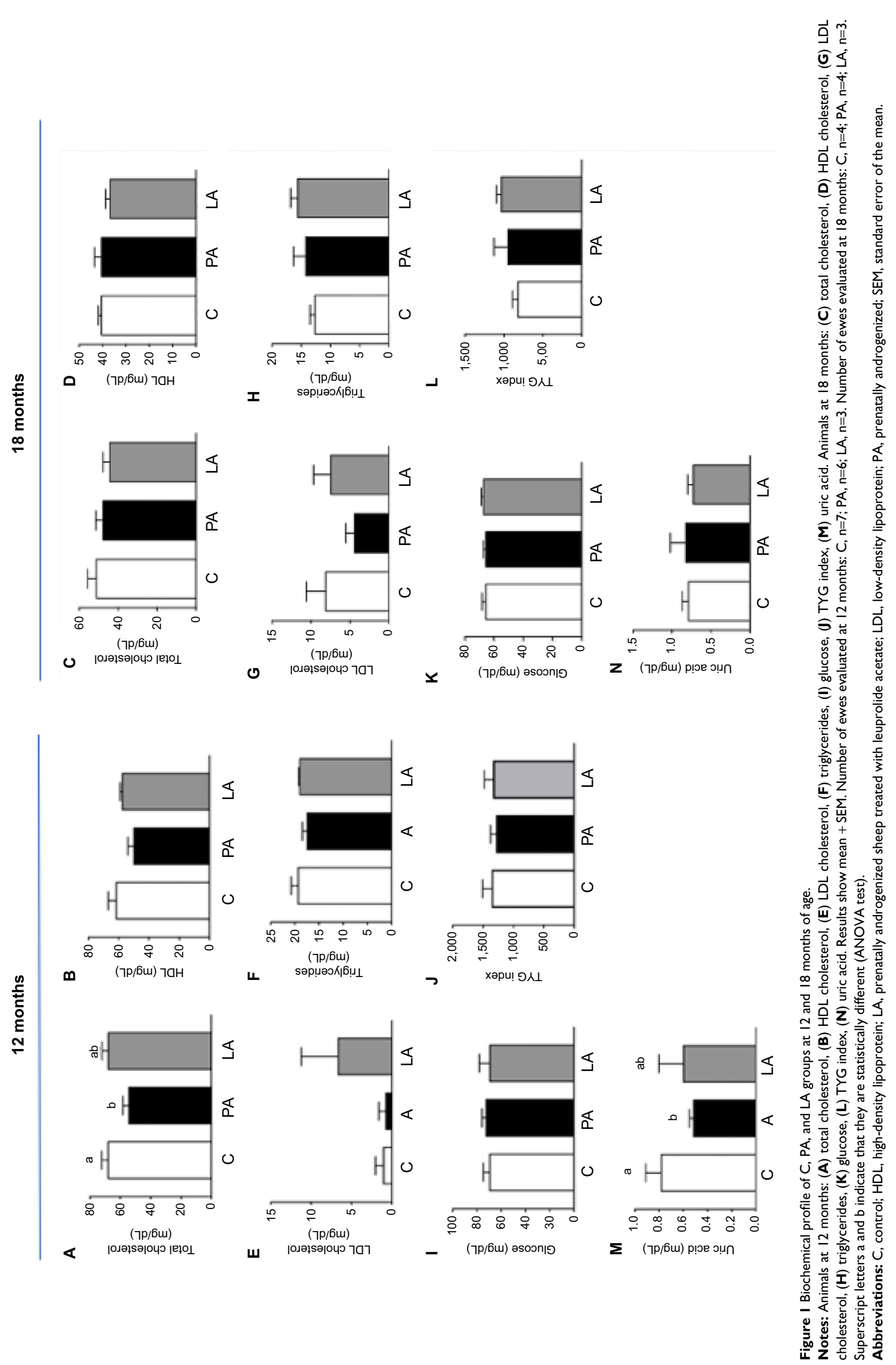


\section{A}

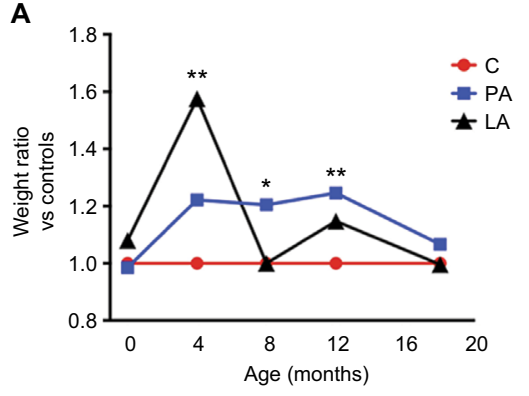

C

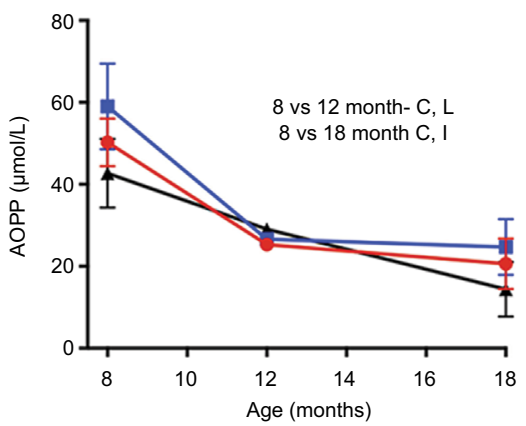

E

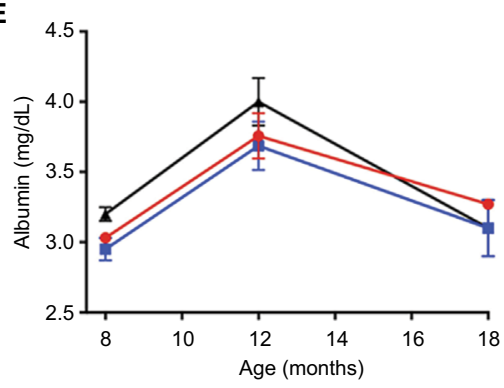

G

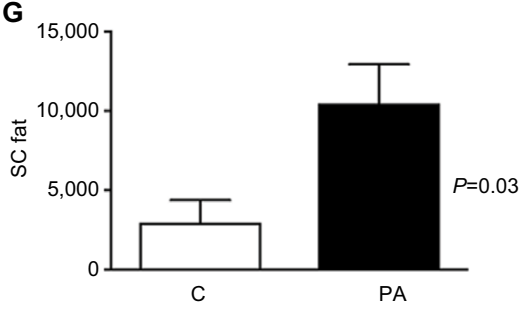

I

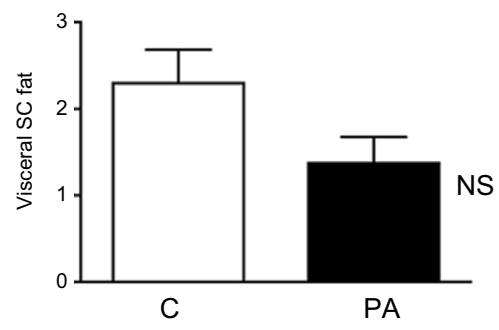

B

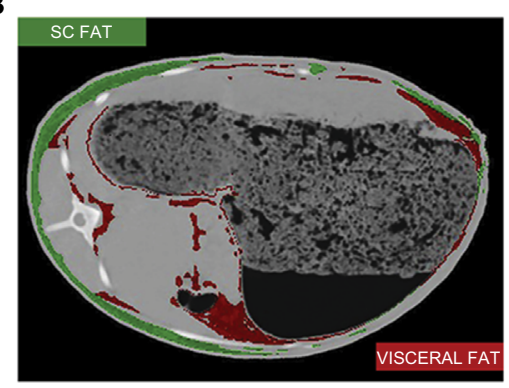

D

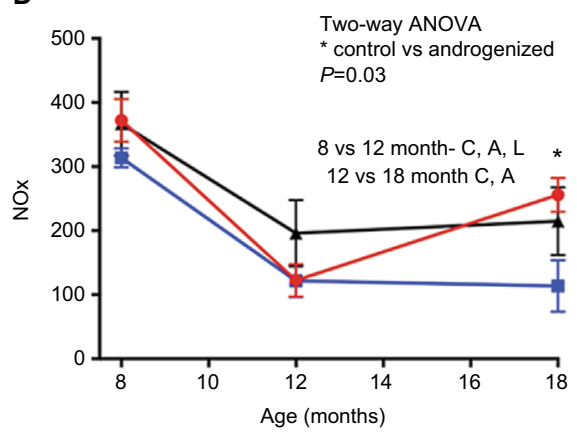

$\mathbf{F}$

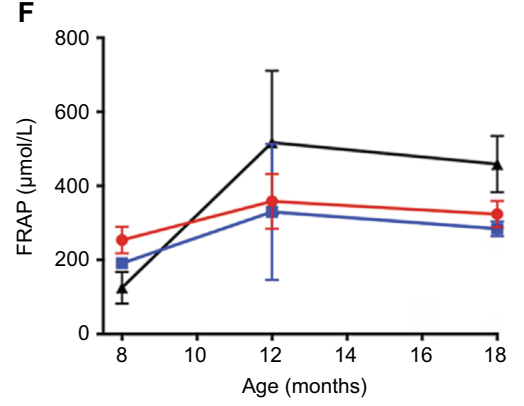

H
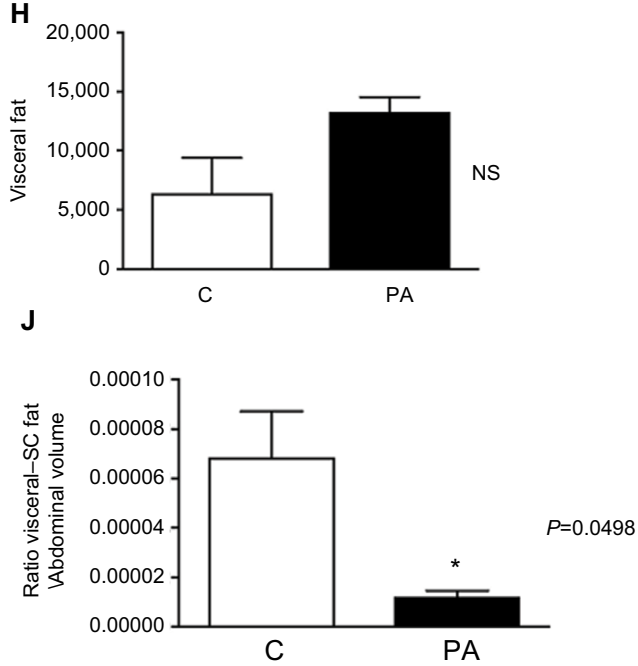

Figure 2 Weight, oxidative stress markers, and fat depot analysis (CT).

Notes: (A) Ratio of weight of C (red), PA sheep (blue), and LA sheep (black). (B) CT image of an abdominal section; images in red and green identify (with the support of the software Analyze 12.0) subcutaneous and visceral fat depots, respectively. Measures of AOPP (C), NOx (D), albumin (E), and FRAP (F) staring from 8th to I8th month of age in C sheep (red), PA sheep (blue), and LA sheep (black). Values of (G) subcutaneous fat mass and (H) visceral fat, both obtained by the use of Analyze I2.0 software. (I) Ratio of visceral to subcutaneous fat and (J) ratio of visceral to subcutaneous fat adjusted to the abdominal area. Results (C-J) show mean \pm SEM. *Indicates statistical significance of weight in kilograms with $P<0.05$ (ANOVA). **P<00I.

Abbreviations: AOPP, advanced oxidation products of proteins; C, control; CT, computed tomography; FRAP, ferric-reducing antioxidant power; LA, prenatally androgenized sheep treated with leuprolide acetate; NOx, nitric oxide; NS, nonsignificant; PA, prenatally androgenized; SC, subcutaneous; SEM, standard error of the mean. 
$329.8 \pm 184$, and $517 \pm 194 \mu \mathrm{mol} / \mathrm{L}$, respectively. C, PA, and LA ewes at 18 months showed no differences in FRAP levels, with values of $323.4 \pm 35.8,284 \pm 20$, and $459 \pm 76.07 \mu \mathrm{mol} / \mathrm{L}$ in the C, PA, and LA groups, respectively (Figure 2F). NOx levels showed a slight decrease from month 8 to 18 of age in the sheep. The initial values of NOx at month 8 were $372 \pm 33.4,313.7 \pm 14.5$, and $368.8 \pm 50$ in the C, PA, and LA animals, respectively (Figure 2D). These values decreased at month 12 in the three groups $(123 \pm 25.2,121.7 \pm 25.2$, and $196 \pm 52$ in the C, PA, and LA groups, respectively). Remarkably, the NOx values at month 18 were significantly decreased in PA ewes compared to those in $\mathrm{C}$ ewes. The NOx levels were 255.9 \pm 26.4 and $113.6 \pm 40$ in the $C$ and PA groups, respectively (two-way ANOVA; $P=0.048$ ). Albumin, an antioxidant scavenger molecule, was also evaluated (Figure 2E). Albumin levels were increased in the three groups at 12 months. At month 18, the albumin levels were 3.27 \pm 0.04 , $3.1 \pm 0.2$, and $3.1 \pm 0.2 \mathrm{mg} / \mathrm{dL}$ in the $\mathrm{C}, \mathrm{PA}$, and LA groups, respectively (Figure $2 \mathrm{E}$ ).

\section{Discussion}

The results of this study revealed a limited impact of GnRH agonist (leuprolide acetate) on modifying metabolic and oxidative stress parameters in PA sheep.

As shown above, neonatal treatment with androgens led to reduced levels of NOx at 18 months of age, which was prevented by administration of LA. NOx is produced by endothelial cells and acts as a second messenger to mediate the inhibition of platelet aggregation and anti-inflammatory and vasodilatation functions. We evaluated the major stable metabolites of NOx, particularly nitrites and nitrates (NOx). NOx has various other functions in reproduction, cardiac function, and cellular signaling.

Although LA improved NOx levels, the treatment did not modify the levels of other oxidative stress markers (FRAP and AOPP) or albumin, glucose, total cholesterol (and its fractions), uric acid, or TYG index. Our results partially agree with those obtained for sheep postnatally treated for 8 weeks with flutamide (an antiandrogen), which did not modify the levels of protein-bound oxidized tyrosine moiety NY, an oxidative stress marker in the plasma. ${ }^{16}$ Our results agree with those of a study in which androgenized rats were treated with LA depot on day 5 of life; although reproductive function was improved, androgens (testosterone or androstenedione) or oxidant and antioxidant molecules in the serum and plasma were not modified. ${ }^{18}$ In the previous study, however, disruption of oxidative stress markers was associated with a worse metabolic profile (TYG) in female
Wistar rats androgenized after birth, which was not observed in our study using sheep. However, as recently described, PA and LA sheep showed significantly elevated insulin levels in an intravenous glucose tolerance test, suggesting insulin resistance in both androgenized groups. ${ }^{29}$

Androgen levels or the presence of hyperandrogenemia also reflects changes in the oxidative stress status, as gender is thought to influence disruptions in the oxidative/antioxidative balance. However, treatment with LA did not change the androgens levels (data not shown). ${ }^{29}$

Interestingly, despite higher weights at 12 months of age, PA sheep exhibited a proportionally lower ratio of visceral to SC fat (estimated by 3D CT) and lower total cholesterol. These features were similar to those described by VeigaLopez et al who reported an increased total amount of visceral fat and reduced ratio of visceral to SC fat in sheep treated with high levels of bisphenol A during gestation. ${ }^{24}$

We evaluated three REDOX markers in an animal model (sheep), which replicate several abnormalities of PCOS and which were followed from month 8 to 18 of life. One limitation of this study was the small number of animals employed (increased chance of beta error). Additionally, we did not evaluate the levels of adipokines (adiponectin, leptin, irisin), which would help to clarify the effects of these molecules on overall inflammation.

In summary, our findings indicate that administration of LA had minimal effects on the REDOX balance and metabolic parameters in PA sheep.

\section{Conclusion}

Our results confirm the limited impact of reproductive drugs such as leuprolide acetate (a GnRH agonist) on markers of the oxidative stress balance.

\section{Ethics approval}

All procedures were approved by the Institutional Committee for Ethics in Animal Experiments at the Federal University of Santa Maria, RS, Brazil (CEUA-UFSM number 0009/2013).

\section{Acknowledgments}

We would like to thank Dr Michael Philcock, Mayo Clinic (Analyze 12.0 software license) and Prof João Francisco Coelho de Oliveira (in memoriam) for his great contribution to the design of the study. This work was supported by CAPES Foundation and the National Council for Scientific and Technological Development (CNPq) Brazil, grant 445019/2014-0 (http://www.cnpq.br/). 


\section{Disclosure}

The authors report no conflicts of interest in this work.

\section{References}

1. Liu Z, Ren Z, Zhang J, et al. Role of ROS and nutritional antioxidants in human diseases. Front Physiol. 2018;9:477.

2. Jiang L, Diaz PT, Best TM, Stimpfl JN, He F, Zuo L. Molecular characterization of redox mechanisms in allergic asthma. Ann Allergy Asthma Immunol. 2014;113(2):137-142.

3. Singh R, Devi S, Gollen R. Role of free radical in atherosclerosis, diabetes and dyslipidaemia: larger-than-life. Diabetes Metab Res Rev. 2015;31(2):113-126.

4. Franks S. Polycystic ovary syndrome. N Engl J Med. 1995;333(13): 853-861.

5. Norman RJ, Dewailly D, Legro RS, Hickey TE. Polycystic ovary syndrome. Lancet. 2007;370(9588):685-697.

6. Balen A, Homburg R, Franks S. Defining polycystic ovary syndrome. BMJ. 2009;338:a2968.

7. Diamanti-Kandarakis E, Argyrakopoulou G, Economou F, Kandaraki E, Koutsilieris M. Defects in insulin signaling pathways in ovarian steroidogenesis and other tissues in polycystic ovary syndrome (PCOS). J Steroid Biochem Mol Biol. 2008;109(3-5):242-246.

8. Deepika ML, Nalini S, Maruthi G, et al. Analysis of oxidative stress status through Mn test and serum MDA levels in PCOS women. PakJ Biol Sci. 2014;17(4):574-577.

9. Azziz R, Carmina E, Chen Z, et al. Polycystic ovary syndrome. Nat Rev Dis Primers. 2016;2:16057.

10. Victor VM, Rovira-Llopis S, Bañuls C, et al. Insulin resistance in PCOS patients enhances oxidative stress and leukocyte adhesion: role of myeloperoxidase. PLoS One. 2016;11(3):e0151960.

11. Abruzzese GA, Cerrone GE, Gamez JM, et al. Lipid accumulation product (LAP) and visceral adiposity index (VAI) as markers of insulin resistance and metabolic associated disturbances in young Argentine women with polycystic ovary syndrome. Horm Metab Res. 2017;49(1):23-29.

12. Bañuls C, Rovira-Llopis S, Martinez de Marañon A, et al. Metabolic syndrome enhances endoplasmic reticulum, oxidative stress and leukocyte-endothelium interactions in PCOS. Metabolism. 2017;71: 153-162.

13. Behboudi-Gandevani S, Amiri M, Bidhendi Yarandi R, et al. The risk of metabolic syndrome in polycystic ovary syndrome: a systematic review and meta-analysis. Clin Endocrinol (Oxf). 2018;88(2):169-184.

14. Zhang R, Liu H, Bai H, et al. Oxidative stress status in Chinese women with different clinical phenotypes of polycystic ovary syndrome. Clin Endocrinol (Oxf). 2017;86(1):88-96.

15. Zhang J, Zhang Y, Liu H, et al. Antioxidant properties of high-density lipoproteins are impaired in women with polycystic ovary syndrome. Fertil Steril. 2015;103(5):1346-1354.
16. Puttabyatappa M, Andriessen V, Mesquitta M, Zeng L, Pennathur S, Padmanabhan V. Developmental programming: impact of gestational steroid and metabolic milieus on mediators of insulin sensitivity in prenatal testosterone-treated female sheep. Endocrinology. 2017;158(9):2783-2798.

17. Tata B, Mimouni NEH, Barbotin AL, et al. Elevated prenatal antiMüllerian hormone reprograms the fetus and induces polycystic ovary syndrome in adulthood. Nat Med. 2018;24(6):834-846.

18. Serrano Mujica LK, Bertolin K, Bridi A, et al. The impact of postnatal leuprolide acetate treatment on reproductive characteristics in a rodent model of polycystic ovary syndrome. Mol Cell Endocrinol. 2017;442:125-133.

19. Miranda VO, Oliveira FC, Dias JH, et al. Estrus resynchronization in ewes with unknown pregnancy status. Theriogenology. 2018;106:103-107.

20. Ortega HH, Rey F, Velazquez MM, Padmanabhan V. Developmental programming: effect of prenatal steroid excess on intraovarian components of insulin signaling pathway and related proteins in sheep. Biol Reprod. 2010;82(6):1065-1075.

21. Benzie IF, Strain JJ. The ferric reducing ability of plasma (FRAP) as a measure of "antioxidant power": the FRAP assay. Anal Biochem. 1996;239(1):70-76.

22. Medeiros MS, Schumacher-Schuh A, Cardoso AM, et al. Iron and oxidative stress in Parkinson's disease: an observational study of injury biomarkers. PLoS One. 2016;11(1):e0146129.

23. Tatsch E, Bochi GV, Pereira RS, et al. A simple and inexpensive automated technique for measurement of serum nitrite/nitrate. Clin Biochem. 2011;44(4):348-350.

24. Veiga-Lopez A, Moeller J, Sreedharan R, et al. Developmental programming: interaction between prenatal BPA exposure and postnatal adiposity on metabolic variables in female sheep. Am J Physiol Endocrinol Metab. 2016;310(3):E238-E247.

25. Simental-Mendía LE, Rodríguez-Morán M, Guerrero-Romero F. The product of fasting glucose and triglycerides as surrogate for identifying insulin resistance in apparently healthy subjects. Metab Syndr Relat Disord. 2008;6(4):299-304.

26. González-Torres L, Vázquez-Velasco M, Olivero-David R, et al. Glucomannan and glucomannan plus Spirulina added to pork significantly block dietary cholesterol effects on lipoproteinemia, arylesterase activity, and CYP7A1 expression in Zucker fa/fa rats. J Physiol Biochem. 2015;71(4): 773-784.

27. Pinto BA, Melo TM, Flister KF, et al. Early and sustained exposure to high-sucrose diet triggers hippocampal ER stress in young rats. Metab Brain Dis. 2016;31(4):917-927.

28. Qu C, Zhou X, Yang G, Li L, Liu H, Liang Z. The natural logarithm of zinc- $\alpha 2$-glycoprotein/HOMA-IR is a better predictor of insulin sensitivity than the product of triglycerides and glucose and the other lipid ratios. Cytokine. 2016;79:96-102.

29. Tonellotto dos Santos J, Escarião da Nóbrega J, Serrano Mujica LK, et al. Prenatal androgenization of ewes as a model of hirsutism in polycystic ovary syndrome. Endocrinology. 2018;159(12):4056-4064.
Journal of Inflammation Research

\section{Publish your work in this journal}

The Journal of Inflammation Research is an international, peer-reviewed open access journal that welcomes laboratory and clinical findings on the molecular basis, cell biology and pharmacology of inflammation including original research, reviews, symposium reports, hypothesis formation and commentaries on: acute/chronic inflammation; mediators of
Dovepress

inflammation; cellular processes; molecular mechanisms; pharmacology and novel anti-inflammatory drugs; clinical conditions involving inflammation. The manuscript management system is completely online and includes a very quick and fair peer-review system. Visit http://www.dove press.com/testimonials.php to read real quotes from published authors. 